Acta Crystallographica Section E

Structure Reports

Online

ISSN 1600-5368

\section{4-Bromomethyl-6-tert-butyl-2H- chromen-2-one}

\section{H. Nagarajaiah, K. B. Puttaraju, K. Shivashankar and Noor Shahina Begum*}

Department of Studies in Chemistry, Bangalore University, Bangalore 560 001, India Correspondence e-mail: noorsb@rediffmail.com,noorsb05@gmail.com

Received 17 May 2013; accepted 4 June 2013

Key indicators: single-crystal X-ray study; $T=100 \mathrm{~K}$; mean $\sigma(\mathrm{C}-\mathrm{C})=0.004 \AA$;

$R$ factor $=0.041 ; w R$ factor $=0.107 ;$ data-to-parameter ratio $=19.0$.

In the crystal structure of the title compound, $\mathrm{C}_{14} \mathrm{H}_{15} \mathrm{BrO}_{2}$, weak $\mathrm{C}-\mathrm{H} \cdots \mathrm{O}$ interactions link the molecules into zigzag chains extending along the $c$-axis direction. These chains are further assembled into (100) layers via $\pi-\pi$ stacking interactions between inversion-related chromenone fragments [interplanar distance $=3.376(2) \AA$ ] .

\section{Related literature}

For therapeutic properties of coumarin derivatives, see: Lacy \& O'Kennedy (2004); Mustafa et al. (2011). For structural features of coumarins, see: Moorthy et al. (2003). For related structures, see: Gowda et al. (2010); Fun et al. (2011).

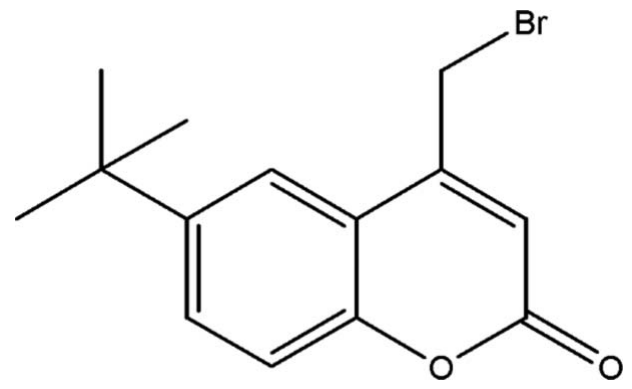

\section{Experimental}

Crystal data

$\mathrm{C}_{14} \mathrm{H}_{15} \mathrm{BrO}_{2}$

$M_{r}=295.17$

Monoclinic, $P 2_{1} / c$ $\beta=97.518(3)^{\circ}$

$V=1264.8(4) \AA^{3}$

$Z=4$

Mo $K \alpha$ radiation

$\mu=3.24 \mathrm{~mm}^{-1}$

$T=100 \mathrm{~K}$

$0.18 \times 0.16 \times 0.16 \mathrm{~mm}$

Data collection

Bruker SMART APEX CCD diffractometer

Absorption correction: multi-scan (SADABS; Bruker, 1998)

$T_{\min }=0.593, T_{\max }=0.625$

Refinement

$R\left[F^{2}>2 \sigma\left(F^{2}\right)\right]=0.041$

$w R\left(F^{2}\right)=0.107$

$S=1.05$

2737 reflections

$\Delta \rho_{\min }=-0.36{\mathrm{e} \AA^{-3}}^{-3}$
7522 measured reflections 2737 independent reflections 2074 reflections with $I>2 \sigma(I)$ $R_{\text {int }}=0.040$

144 parameters

$\mathrm{H}$-atom parameters constrained $\Delta \rho_{\max }=0.78{\mathrm{e} \AA^{-3}}^{-3}$

Table 1

Hydrogen-bond geometry $\left(\AA,^{\circ}\right)$.

\begin{tabular}{lllll}
\hline$D-\mathrm{H} \cdots A$ & $D-\mathrm{H}$ & $\mathrm{H} \cdots A$ & $D \cdots A$ & $D-\mathrm{H} \cdots A$ \\
\hline $\mathrm{C} 3-\mathrm{H} 3 \cdots \mathrm{O} 2{ }^{\mathrm{i}}$ & 0.95 & 2.42 & $3.334(4)$ & 162 \\
\hline
\end{tabular}

Symmetry code: (i) $x,-y+\frac{1}{2}, z-\frac{1}{2}$.

Data collection: SMART (Bruker,1998); cell refinement: SAINTPlus (Bruker,1998); data reduction: SAINT-Plus; program(s) used to solve structure: SHELXS97 (Sheldrick, 2008); program(s) used to refine structure: SHELXL97 (Sheldrick, 2008); molecular graphics: ORTEP-3 for Windows (Farrugia, 2012) and CAMERON (Watkin et al., 1996); software used to prepare material for publication: Win $G X$ (Farrugia, 2012).

NSB and KSS are thankful to the University Grants Commission (UGC), India, for financial assistance. HN and PKB thank UGC for fellowships.

Supplementary data and figures for this paper are available from the IUCr electronic archives (Reference: GK2575).

\title{
References
}

Bruker. (1998). SMART, SAINT-Plus and SADABS. Bruker Axs Inc., Madison, Wisconcin, USA.

Farrugia, L. J. (2012). J. Appl. Cryst. 45, 849-854.

Fun, H.-K., Goh, J. H., Wu, D. \& Zhang, Y. (2011). Acta Cryst. E67, o136.

Gowda, R., Basanagouda, M., Kulkarni, M. V. \& Gowda, K. V. A. (2010). Acta Cryst. E66, o2906.

Lacy, A. \& O’Kennedy, R. (2004). Curr. Pharm. Des. 10, 3797-3811.

Moorthy, J. N., Venkatakrishnan, P. \& Singh, A. S. (2003). CrystEngComm, 5 507-513.

Mustafa, M. S., El-Abadelah, M. M., Zihlif, M. A., Naffa, R. G. \& Mubarak, M. S. (2011). Molecules, 16, 4305-4317.

Sheldrick, G. M. (2008). Acta Cryst. A64, 112-122.

Watkin, D. J., Prout, C. K. \& Pearce, L. J. (1996). CAMERON. Chemical Crystallography Laboratory, University of Oxford, England. 


\section{supporting information}

Acta Cryst. (2013). E69, o1056 [https://doi.org/10.1107/S1600536813015511]

\section{4-Bromomethyl-6-tert-butyl-2H-chromen-2-one}

\section{H. Nagarajaiah, K. B. Puttaraju, K. Shivashankar and Noor Shahina Begum}

\section{S1. Comment}

Coumarins are of great interest due to their biological properties (Lacy \& O'Kennedy 2004). In particular, their physiological, bacteriostatic and anti-tumour activity (Mustafa et al., 2011) makes these compounds attractive for further backbone derivatization and screening for their therapeutic properties.

In the title compound, $\mathrm{C}_{15} \mathrm{H}_{14} \mathrm{BrO}_{2}$ (Fig. 1), the coumarin ring is substituted with bromomethyl group at $\mathrm{C} 4$ and tertbutyl group at C6. The coumarin ring is essentialy planar (r.m.s. deviation $=0.019 \AA$ ). Among the three methyl groups belonging to tert-butyl moiety two methyl groups, $\mathrm{C} 12$ \& $\mathrm{C} 13$, deviate from the plane of the coumarin ring whereas the carbon atom $\mathrm{C} 14$ of the methyl group lies within the plane. The crystal structure is stabilized by $\mathrm{C}-\mathrm{H} \cdots \mathrm{O}$ interactions (Moorthy et al. 2003). The $\mathrm{C} 3-\mathrm{H} 3 \cdots \mathrm{O} 2$ interaction results in zigzag chains running along the $c$-axis (Fig. 2). There are intermolecular $\pi-\pi$ interactions between two anti-parallel molecules in the unit cell with an interplanar distance of 3.376 (2) Å. For crystal structures related to the title compound, see: Gowda et al. (2010); Fun et al. (2011).

\section{S2. Experimental}

To a mixture of equimolar quantity of 4-tert-butyl phenol $(0.1 \mathrm{~mol})$ and 4-bromoethyl acetoacetate $(0.1 \mathrm{~mol})$ was added dropwise Conc. sulfuric acid $(30 \mathrm{ml})$ with constant stirring and maintaining the temperature between $273-278 \mathrm{~K}$. The reaction mixture was allowed to stand in ice chest overnight and deep red coloured solution was poured into the stream of crushed ice. Solid separated was filtered and washed with water and then with cold ethanol so as to get a colourless compound. Finally, it was recrystallized from ethyl acetate. Yield 89\%; colorless solid; m.p. 417-420 K; IR (KBr, cm-1): 1700 (lactone $\mathrm{C}=\mathrm{O}$ ), ${ }^{1} \mathrm{H}$ NMR (300 MHz, DMSO-d 6 ): $\delta 1.32$ (s, 9H, 6-tert-butyl), 4.93 (s, 2H, CH2-Br), 6.70 (s, 1H, C3-H), 7.34 (d, 1H, C7-H, $J=6.2 \mathrm{~Hz}$ ), 7.68 (d, 1H, C8-H, $J=8.1 \mathrm{~Hz}), 7.80$ (s, 1H, C5-H): LC-MS 297 [M+ 2]: Anal. Cald. for $\mathrm{C}_{15} \mathrm{H}_{14} \mathrm{Br}_{1} \mathrm{O}_{2}$ : C 56.97; H 5.12. Found: C 56.91; H 5.04.

\section{S3. Refinement}

The $\mathrm{H}$ atoms were placed at calculated positions in the riding model approximation with $\mathrm{C}-\mathrm{H}=0.95,0.98$, and $0.99 \AA$ for aryl, methyl, and methylene $\mathrm{H}$-atoms respectively, with $U_{\text {iso }}(\mathrm{H})=1.5 U_{\text {eq }}(\mathrm{C})$ for methyl $\mathrm{H}$ atoms and $U_{\text {iso }}(\mathrm{H})=$ $1.2 U_{\mathrm{eq}}(\mathrm{C})$ for other $\mathrm{H}$ atom. 


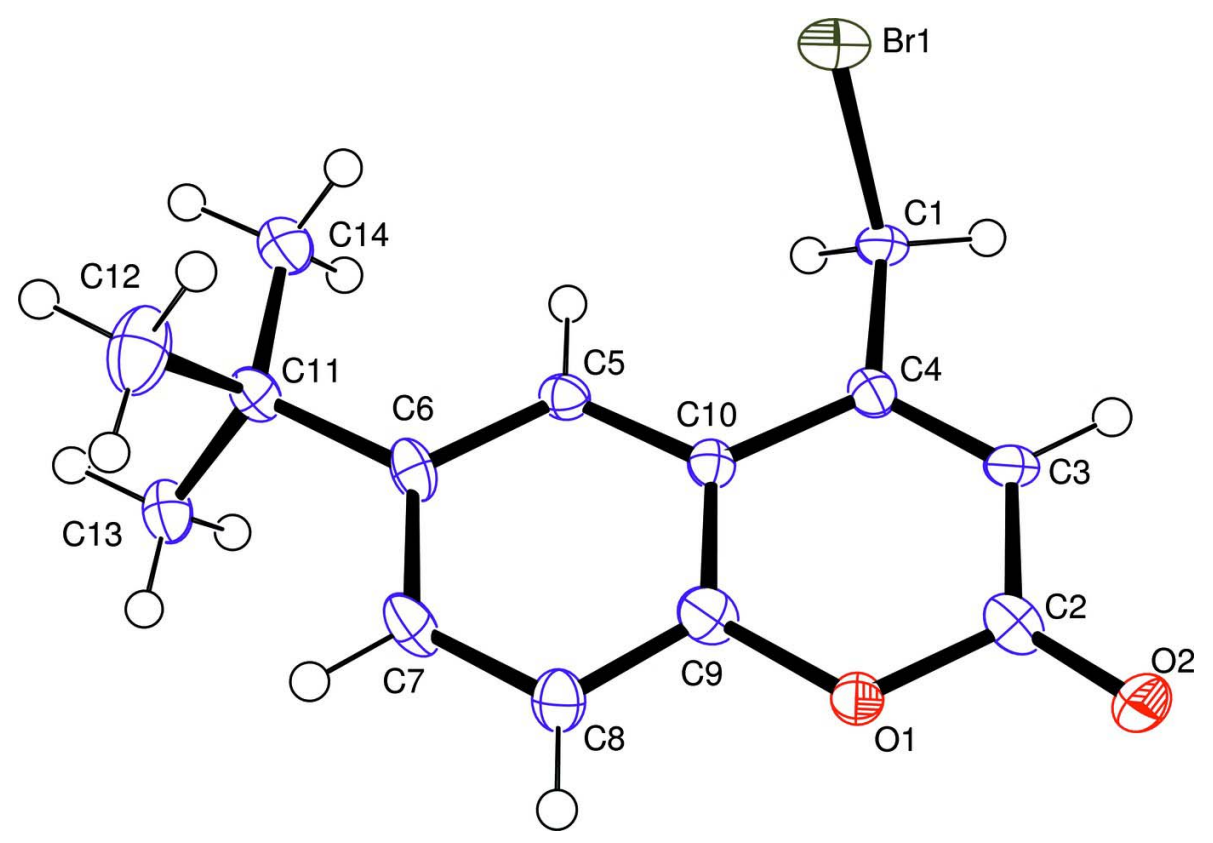

Figure 1

Molecular structure of the title compound showing $50 \%$ probability ellipsoids. 


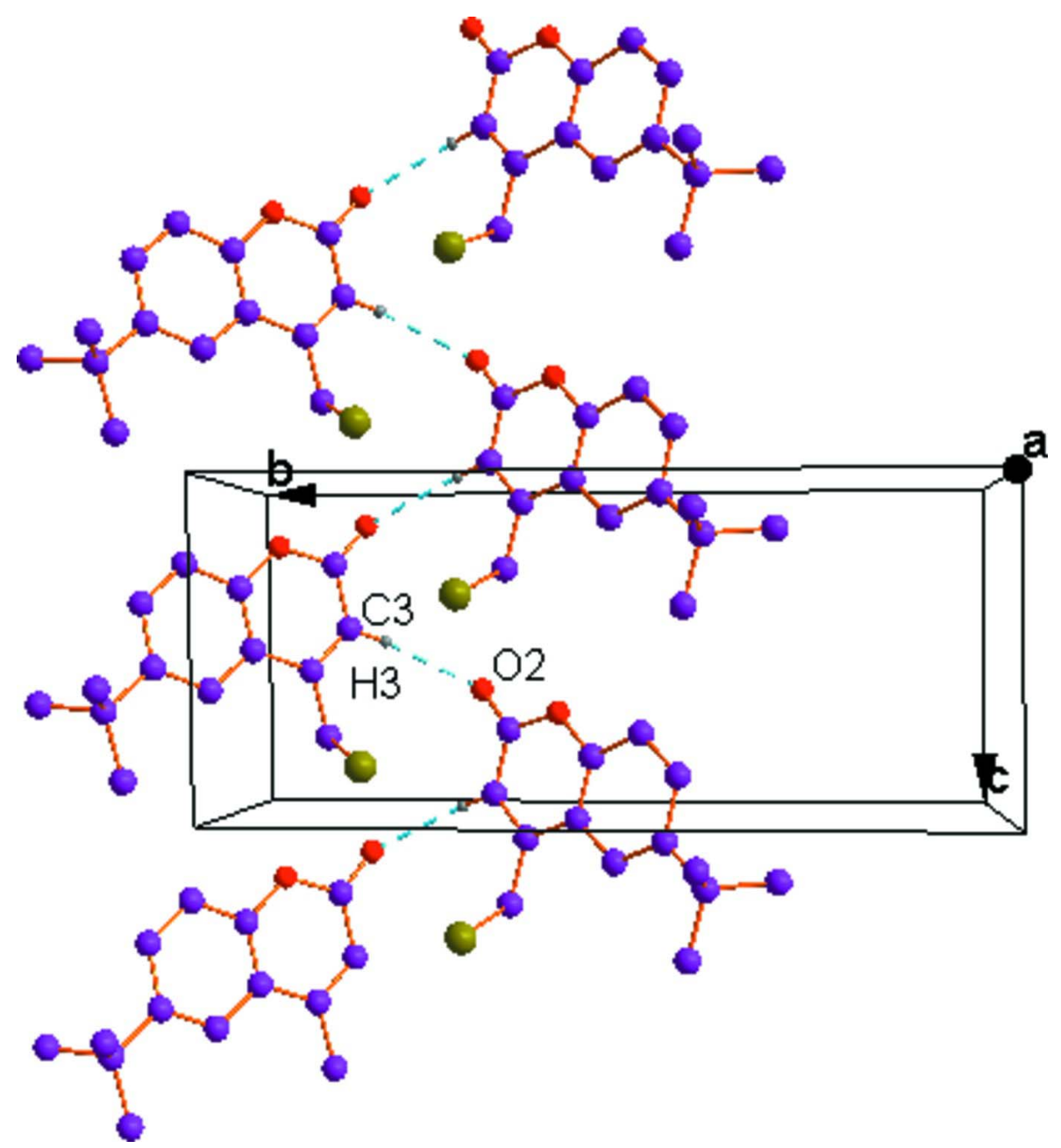

Figure 2

Chains of molecules formed by $\mathrm{C}-\mathrm{H} \cdots \mathrm{O}$ interaction. Dotted lines indicate intermolecular interactions. $\mathrm{H}$-atoms not involved in hydrogen bonding have been excluded.

\section{4-Bromomethyl-6-tert-butyl-2H-chromen-2-one}

\section{Crystal data}

$\mathrm{C}_{14} \mathrm{H}_{15} \mathrm{BrO}_{2}$

$M_{r}=295.17$

Monoclinic, $P 2_{1} / c$

Hall symbol: $-\mathrm{P} 2 \mathrm{ybc}$

$a=10.3311(19) \AA$

$b=16.830(3) \AA$

$c=7.3374$ (14) $\AA$

$\beta=97.518(3)^{\circ}$

$V=1264.8(4) \AA^{3}$

$Z=4$
$F(000)=600$

$D_{\mathrm{x}}=1.550 \mathrm{Mg} \mathrm{m}^{-3}$

Mo $K \alpha$ radiation, $\lambda=0.71073 \AA$

Cell parameters from 2074 reflections

$\theta=2.3-27.0^{\circ}$

$\mu=3.24 \mathrm{~mm}^{-1}$

$T=100 \mathrm{~K}$

Block, colourless

$0.18 \times 0.16 \times 0.16 \mathrm{~mm}$ 


\section{Data collection}

Bruker SMART APEX CCD

diffractometer

Radiation source: fine-focus sealed tube

Graphite monochromator

$\omega$ scans

Absorption correction: multi-scan

(SADABS; Bruker, 1998)

$T_{\text {min }}=0.593, T_{\max }=0.625$

\section{Refinement}

Refinement on $F^{2}$

Least-squares matrix: full

$R\left[F^{2}>2 \sigma\left(F^{2}\right)\right]=0.041$

$w R\left(F^{2}\right)=0.107$

$S=1.05$

2737 reflections

144 parameters

0 restraints

Primary atom site location: structure-invariant direct methods
7522 measured reflections

2737 independent reflections

2074 reflections with $I>2 \sigma(I)$

$R_{\text {int }}=0.040$

$\theta_{\max }=27.0^{\circ}, \theta_{\min }=2.3^{\circ}$

$h=-13 \rightarrow 12$

$k=-21 \rightarrow 16$

$l=-9 \rightarrow 9$

Secondary atom site location: difference Fourier map

Hydrogen site location: inferred from

neighbouring sites

$\mathrm{H}$-atom parameters constrained

$w=1 /\left[\sigma^{2}\left(F_{0}^{2}\right)+(0.0531 P)^{2}\right]$

where $P=\left(F_{\mathrm{o}}^{2}+2 F_{\mathrm{c}}^{2}\right) / 3$

$(\Delta / \sigma)_{\max }<0.001$

$\Delta \rho_{\max }=0.78$ e $\AA^{-3}$

$\Delta \rho_{\min }=-0.36$ e $\AA^{-3}$

Special details

Geometry. All esds (except the esd in the dihedral angle between two 1.s. planes) are estimated using the full covariance matrix. The cell esds are taken into account individually in the estimation of esds in distances, angles and torsion angles; correlations between esds in cell parameters are only used when they are defined by crystal symmetry. An approximate (isotropic) treatment of cell esds is used for estimating esds involving l.s. planes.

Refinement. Refinement of $F^{2}$ against ALL reflections. The weighted $R$-factor $w R$ and goodness of fit $S$ are based on $F^{2}$, conventional $R$-factors $R$ are based on $F$, with $F$ set to zero for negative $F^{2}$. The threshold expression of $F^{2}>2 \sigma\left(F^{2}\right)$ is used only for calculating $R$-factors (gt) etc. and is not relevant to the choice of reflections for refinement. $R$-factors based on $F^{2}$ are statistically about twice as large as those based on $F$, and $R$ - factors based on ALL data will be even larger.

Fractional atomic coordinates and isotropic or equivalent isotropic displacement parameters $\left(\AA^{2}\right)$

\begin{tabular}{lllll}
\hline & $x$ & $y$ & $z$ & $U_{\text {iso }} * / U_{\text {eq }}$ \\
\hline C1 & $0.4032(3)$ & $0.13349(18)$ & $0.2373(4)$ & $0.0198(7)$ \\
H1A & 0.4081 & 0.0849 & 0.1624 & $0.024^{*}$ \\
H1B & 0.4768 & 0.1686 & 0.2164 & $0.024^{*}$ \\
C2 & $0.5105(3)$ & $0.13683(17)$ & $0.7557(4)$ & $0.0190(6)$ \\
C3 & $0.4913(3)$ & $0.15577(18)$ & $0.5616(4)$ & $0.0190(6)$ \\
H3 & 0.5340 & 0.2011 & 0.5205 & $0.023^{*}$ \\
C4 & $0.4148(3)$ & $0.11146(17)$ & $0.4361(4)$ & $0.0173(6)$ \\
C5 & $0.2620(3)$ & $-0.00601(18)$ & $0.3828(4)$ & $0.0171(6)$ \\
H5 & 0.2467 & 0.0057 & 0.2551 & $0.021^{*}$ \\
C6 & $0.1996(3)$ & $-0.07068(17)$ & $0.4490(4)$ & $0.0187(6)$ \\
C7 & $0.2270(3)$ & $-0.08765(18)$ & $0.6383(4)$ & $0.0215(7)$ \\
H7 & 0.1870 & -0.1324 & 0.6869 & $0.026^{*}$ \\
C8 & $0.3104(3)$ & $-0.04076(18)$ & $0.7543(4)$ & $0.0215(7)$ \\
H8 & 0.3276 & -0.0530 & 0.8816 & $0.026^{*}$ \\
C9 & $0.3687(3)$ & $0.02395(17)$ & $0.6843(4)$ & $0.0182(6)$ \\
C10 & $0.3472(3)$ & $0.04295(17)$ & $0.4984(4)$ & $0.0161(6)$
\end{tabular}




$\begin{array}{lllll}\text { C11 } & 0.1007(3) & -0.12192(18) & 0.3272(4) & 0.0210(7) \\ \text { C12 } & -0.0343(3) & -0.11188(14) & 0.3930(5) & 0.0368(9) \\ \text { H12A } & -0.0993 & -0.1429 & 0.3137 & 0.055^{*} \\ \text { H12B } & -0.0590 & -0.0556 & 0.3871 & 0.055^{*} \\ \text { H12C } & -0.0301 & -0.1307 & 0.5200 & 0.055^{*} \\ \text { C13 } & 0.1402(3) & -0.21004(14) & 0.3427(4) & 0.0265(7) \\ \text { H13A } & 0.2251 & -0.2171 & 0.2987 & 0.040^{*} \\ \text { H13B } & 0.0744 & -0.2421 & 0.2680 & 0.040^{*} \\ \text { H13C } & 0.1465 & -0.2269 & 0.4716 & 0.040^{*} \\ \text { C14 } & 0.0902(2) & -0.09867(5) & 0.12412(5) & 0.0305(8) \\ \text { H14A } & 0.1753 & -0.1056 & 0.0808 & 0.046^{*} \\ \text { H14B } & 0.0632 & -0.0430 & 0.1096 & 0.046^{*} \\ \text { H14C } & 0.0255 & -0.1326 & 0.0520 & 0.046^{*} \\ \text { O1 } & 0.45022(6) & 0.06915(5) & 0.80902(6) & 0.0197(5) \\ \text { O2 } & 0.57521(5) & 0.17418(5) & 0.87563(5) & 0.0241(5) \\ \text { Br1 } & 0.23783(3) & 0.18813(2) & 0.16045(4) & 0.03299(15)\end{array}$

Atomic displacement parameters $\left(\AA^{2}\right)$

\begin{tabular}{lllllll}
\hline & $U^{11}$ & $U^{22}$ & $U^{33}$ & $U^{12}$ & $U^{13}$ & $U^{23}$ \\
\hline C1 & $0.0208(16)$ & $0.0200(16)$ & $0.0194(15)$ & $0.0001(12)$ & $0.0053(12)$ & $0.0043(12)$ \\
C2 & $0.0205(16)$ & $0.0150(15)$ & $0.0227(16)$ & $0.0037(12)$ & $0.0073(13)$ & $-0.0024(12)$ \\
C3 & $0.0178(16)$ & $0.0190(16)$ & $0.0205(15)$ & $0.0001(12)$ & $0.0036(12)$ & $0.0029(12)$ \\
C4 & $0.0161(15)$ & $0.0153(15)$ & $0.0208(15)$ & $0.0031(12)$ & $0.0035(12)$ & $0.0013(12)$ \\
C5 & $0.0165(15)$ & $0.0185(16)$ & $0.0164(14)$ & $0.0038(12)$ & $0.0018(12)$ & $0.0026(11)$ \\
C6 & $0.0170(15)$ & $0.0157(15)$ & $0.0246(16)$ & $0.0017(12)$ & $0.0066(12)$ & $-0.0007(12)$ \\
C7 & $0.0251(17)$ & $0.0155(15)$ & $0.0252(16)$ & $0.0007(13)$ & $0.0082(13)$ & $0.0021(12)$ \\
C8 & $0.0257(17)$ & $0.0232(17)$ & $0.0164(15)$ & $0.0013(13)$ & $0.0063(13)$ & $0.0009(12)$ \\
C9 & $0.0189(16)$ & $0.0202(16)$ & $0.0159(14)$ & $0.0016(12)$ & $0.0036(12)$ & $-0.0034(12)$ \\
C10 & $0.0163(15)$ & $0.0146(14)$ & $0.0177(14)$ & $0.0035(11)$ & $0.0038(11)$ & $-0.0021(11)$ \\
C11 & $0.0219(16)$ & $0.0153(15)$ & $0.0260(17)$ & $-0.0002(12)$ & $0.0046(13)$ & $0.0010(13)$ \\
C12 & $0.0241(19)$ & $0.030(2)$ & $0.057(2)$ & $-0.0062(15)$ & $0.0107(17)$ & $-0.0144(18)$ \\
C13 & $0.034(2)$ & $0.0228(17)$ & $0.0230(17)$ & $-0.0016(14)$ & $0.0032(14)$ & $-0.0003(13)$ \\
C14 & $0.033(2)$ & $0.0261(19)$ & $0.0288(18)$ & $-0.0086(15)$ & $-0.0094(15)$ & $0.0047(14)$ \\
O1 & $0.0252(12)$ & $0.0186(11)$ & $0.0149(10)$ & $-0.0026(9)$ & $0.0014(9)$ & $0.0002(8)$ \\
O2 & $0.0283(13)$ & $0.0232(12)$ & $0.0202(11)$ & $-0.0030(9)$ & $0.0010(9)$ & $-0.0037(9)$ \\
Br1 & $0.0305(2)$ & $0.0332(2)$ & $0.0326(2)$ & $0.00355(15)$ & $-0.00600(15)$ & $0.01003(15)$ \\
& & & & & &
\end{tabular}

Geometric parameters $\left(A,{ }^{\circ}\right)$

\begin{tabular}{llll}
\hline $\mathrm{C} 1-\mathrm{C} 4$ & $1.494(4)$ & $\mathrm{C} 8-\mathrm{C} 9$ & $1.376(4)$ \\
$\mathrm{C} 1-\mathrm{Br} 1$ & $1.957(3)$ & $\mathrm{C} 8-\mathrm{H} 8$ & 0.9500 \\
$\mathrm{C} 1-\mathrm{H} 1 \mathrm{~A}$ & 0.9900 & $\mathrm{C} 9-\mathrm{O} 1$ & $1.387(3)$ \\
$\mathrm{C} 1-\mathrm{H} 1 \mathrm{~B}$ & 0.9900 & $\mathrm{C} 9-\mathrm{C} 10$ & $1.390(4)$ \\
$\mathrm{C} 2-\mathrm{O} 2$ & $1.209(3)$ & $\mathrm{C} 11-\mathrm{C} 14$ & $1.531(3)$ \\
$\mathrm{C} 2-\mathrm{O} 1$ & $1.379(3)$ & $\mathrm{C} 11-\mathrm{C} 13$ & $1.539(4)$ \\
$\mathrm{C} 2-\mathrm{C} 3$ & $1.448(4)$ & $\mathrm{C} 11-\mathrm{C} 12$ & $1.544(4)$ \\
$\mathrm{C} 3-\mathrm{C} 4$ & $1.356(4)$ & $\mathrm{C} 12-\mathrm{H} 12 \mathrm{~A}$ & 0.9800
\end{tabular}




\begin{tabular}{|c|c|c|c|}
\hline $\mathrm{C} 3-\mathrm{H} 3$ & 0.9500 & $\mathrm{C} 12-\mathrm{H} 12 \mathrm{~B}$ & 0.9800 \\
\hline $\mathrm{C} 4-\mathrm{C} 10$ & $1.452(4)$ & $\mathrm{C} 12-\mathrm{H} 12 \mathrm{C}$ & 0.9800 \\
\hline $\mathrm{C} 5-\mathrm{C} 6$ & $1.385(4)$ & $\mathrm{C} 13-\mathrm{H} 13 \mathrm{~A}$ & 0.9800 \\
\hline $\mathrm{C} 5-\mathrm{C} 10$ & $1.407(4)$ & C13-H13B & 0.9782 \\
\hline $\mathrm{C} 5-\mathrm{H} 5$ & 0.9500 & $\mathrm{C} 13-\mathrm{H} 13 \mathrm{C}$ & 0.9819 \\
\hline $\mathrm{C} 6-\mathrm{C} 7$ & $1.410(4)$ & $\mathrm{C} 14-\mathrm{H} 14 \mathrm{~A}$ & 0.9800 \\
\hline $\mathrm{C} 6-\mathrm{C} 11$ & $1.532(4)$ & C14-H14B & 0.9800 \\
\hline $\mathrm{C} 7-\mathrm{C} 8$ & $1.378(4)$ & $\mathrm{C} 14-\mathrm{H} 14 \mathrm{C}$ & 0.9800 \\
\hline $\mathrm{C} 7-\mathrm{H} 7$ & 0.9500 & & \\
\hline $\mathrm{C} 4-\mathrm{C} 1-\mathrm{Br} 1$ & $110.7(2)$ & $\mathrm{O} 1-\mathrm{C} 9-\mathrm{C} 10$ & $121.8(3)$ \\
\hline $\mathrm{C} 4-\mathrm{C} 1-\mathrm{H} 1 \mathrm{~A}$ & 109.5 & $\mathrm{C} 9-\mathrm{C} 10-\mathrm{C} 5$ & $117.6(3)$ \\
\hline $\mathrm{Br} 1-\mathrm{C} 1-\mathrm{H} 1 \mathrm{~A}$ & 109.5 & $\mathrm{C} 9-\mathrm{C} 10-\mathrm{C} 4$ & 118.0 \\
\hline $\mathrm{C} 4-\mathrm{C} 1-\mathrm{H} 1 \mathrm{~B}$ & 109.5 & $\mathrm{C} 5-\mathrm{C} 10-\mathrm{C} 4$ & $124.3(3)$ \\
\hline $\mathrm{Br} 1-\mathrm{C} 1-\mathrm{H} 1 \mathrm{~B}$ & 109.5 & $\mathrm{C} 14-\mathrm{C} 11-\mathrm{C} 6$ & $112.4(2)$ \\
\hline $\mathrm{H} 1 \mathrm{~A}-\mathrm{C} 1-\mathrm{H} 1 \mathrm{~B}$ & 108.1 & $\mathrm{C} 14-\mathrm{C} 11-\mathrm{C} 13$ & $107.6(2)$ \\
\hline $\mathrm{O} 2-\mathrm{C} 2-\mathrm{O} 1$ & $116.8(2)$ & $\mathrm{C} 6-\mathrm{C} 11-\mathrm{C} 13$ & $110.4(2)$ \\
\hline $\mathrm{O} 2-\mathrm{C} 2-\mathrm{C} 3$ & $126.4(3)$ & $\mathrm{C} 14-\mathrm{C} 11-\mathrm{C} 12$ & $109.0(2)$ \\
\hline $\mathrm{O} 1-\mathrm{C} 2-\mathrm{C} 3$ & $116.9(2)$ & $\mathrm{C} 6-\mathrm{C} 11-\mathrm{C} 12$ & $108.4(2)$ \\
\hline $\mathrm{C} 4-\mathrm{C} 3-\mathrm{C} 2$ & $122.7(3)$ & $\mathrm{C} 13-\mathrm{C} 11-\mathrm{C} 12$ & $108.9(2)$ \\
\hline $\mathrm{C} 4-\mathrm{C} 3-\mathrm{H} 3$ & 118.7 & $\mathrm{C} 11-\mathrm{C} 12-\mathrm{H} 12 \mathrm{~A}$ & 109.5 \\
\hline $\mathrm{C} 2-\mathrm{C} 3-\mathrm{H} 3$ & 118.7 & $\mathrm{C} 11-\mathrm{C} 12-\mathrm{H} 12 \mathrm{~B}$ & 109.5 \\
\hline $\mathrm{C} 3-\mathrm{C} 4-\mathrm{C} 10$ & $119.0(3)$ & $\mathrm{H} 12 \mathrm{~A}-\mathrm{C} 12-\mathrm{H} 12 \mathrm{~B}$ & 109.5 \\
\hline $\mathrm{C} 3-\mathrm{C} 4-\mathrm{C} 1$ & $119.4(3)$ & $\mathrm{C} 11-\mathrm{C} 12-\mathrm{H} 12 \mathrm{C}$ & 109.5 \\
\hline $\mathrm{C} 10-\mathrm{C} 4-\mathrm{C} 1$ & $121.6(3)$ & $\mathrm{H} 12 \mathrm{~A}-\mathrm{C} 12-\mathrm{H} 12 \mathrm{C}$ & 109.5 \\
\hline $\mathrm{C} 6-\mathrm{C} 5-\mathrm{C} 10$ & $122.1(3)$ & $\mathrm{H} 12 \mathrm{~B}-\mathrm{C} 12-\mathrm{H} 12 \mathrm{C}$ & 109.5 \\
\hline $\mathrm{C} 6-\mathrm{C} 5-\mathrm{H} 5$ & 118.9 & $\mathrm{C} 11-\mathrm{C} 13-\mathrm{H} 13 \mathrm{~A}$ & 109.5 \\
\hline $\mathrm{C} 10-\mathrm{C} 5-\mathrm{H} 5$ & 118.9 & $\mathrm{C} 11-\mathrm{C} 13-\mathrm{H} 13 \mathrm{~B}$ & 109.4 \\
\hline $\mathrm{C} 5-\mathrm{C} 6-\mathrm{C} 7$ & $117.6(3)$ & $\mathrm{H} 13 \mathrm{~A}-\mathrm{C} 13-\mathrm{H} 13 \mathrm{~B}$ & 109.5 \\
\hline $\mathrm{C} 5-\mathrm{C} 6-\mathrm{C} 11$ & $122.9(3)$ & $\mathrm{C} 11-\mathrm{C} 13-\mathrm{H} 13 \mathrm{C}$ & 109.5 \\
\hline $\mathrm{C} 7-\mathrm{C} 6-\mathrm{C} 11$ & $119.6(3)$ & $\mathrm{H} 13 \mathrm{~A}-\mathrm{C} 13-\mathrm{H} 13 \mathrm{C}$ & 109.4 \\
\hline $\mathrm{C} 8-\mathrm{C} 7-\mathrm{C} 6$ & $121.4(3)$ & $\mathrm{H} 13 \mathrm{~B}-\mathrm{C} 13-\mathrm{H} 13 \mathrm{C}$ & 109.5 \\
\hline $\mathrm{C} 8-\mathrm{C} 7-\mathrm{H} 7$ & 119.3 & $\mathrm{C} 11-\mathrm{C} 14-\mathrm{H} 14 \mathrm{~A}$ & 109.5 \\
\hline $\mathrm{C} 6-\mathrm{C} 7-\mathrm{H} 7$ & 119.3 & $\mathrm{C} 11-\mathrm{C} 14-\mathrm{H} 14 \mathrm{~B}$ & 109.5 \\
\hline $\mathrm{C} 9-\mathrm{C} 8-\mathrm{C} 7$ & $119.4(3)$ & $\mathrm{H} 14 \mathrm{~A}-\mathrm{C} 14-\mathrm{H} 14 \mathrm{~B}$ & 109.5 \\
\hline $\mathrm{C} 9-\mathrm{C} 8-\mathrm{H} 8$ & 120.3 & $\mathrm{C} 11-\mathrm{C} 14-\mathrm{H} 14 \mathrm{C}$ & 109.5 \\
\hline $\mathrm{C} 7-\mathrm{C} 8-\mathrm{H} 8$ & 120.3 & $\mathrm{H} 14 \mathrm{~A}-\mathrm{C} 14-\mathrm{H} 14 \mathrm{C}$ & 109.5 \\
\hline $\mathrm{C} 8-\mathrm{C} 9-\mathrm{O} 1$ & $116.4(2)$ & $\mathrm{H} 14 \mathrm{~B}-\mathrm{C} 14-\mathrm{H} 14 \mathrm{C}$ & 109.5 \\
\hline $\mathrm{C} 8-\mathrm{C} 9-\mathrm{C} 10$ & $121.8(3)$ & $\mathrm{C} 2-\mathrm{O} 1-\mathrm{C} 9$ & $121.59(17)$ \\
\hline $\mathrm{O} 2-\mathrm{C} 2-\mathrm{C} 3-\mathrm{C} 4$ & $-178.6(3)$ & $\mathrm{C} 6-\mathrm{C} 5-\mathrm{C} 10-\mathrm{C} 9$ & $0.6(4)$ \\
\hline $\mathrm{O} 1-\mathrm{C} 2-\mathrm{C} 3-\mathrm{C} 4$ & $2.0(4)$ & $\mathrm{C} 6-\mathrm{C} 5-\mathrm{C} 10-\mathrm{C} 4$ & $-179.9(3)$ \\
\hline $\mathrm{C} 2-\mathrm{C} 3-\mathrm{C} 4-\mathrm{C} 10$ & $1.1(4)$ & $\mathrm{C} 3-\mathrm{C} 4-\mathrm{C} 10-\mathrm{C} 9$ & $-2.7(4)$ \\
\hline $\mathrm{C} 2-\mathrm{C} 3-\mathrm{C} 4-\mathrm{C} 1$ & $-178.1(3)$ & $\mathrm{C} 1-\mathrm{C} 4-\mathrm{C} 10-\mathrm{C} 9$ & $176.5(3)$ \\
\hline $\mathrm{Br} 1-\mathrm{C} 1-\mathrm{C} 4-\mathrm{C} 3$ & $-102.3(3)$ & $\mathrm{C} 3-\mathrm{C} 4-\mathrm{C} 10-\mathrm{C} 5$ & $177.8(3)$ \\
\hline $\mathrm{Br} 1-\mathrm{C} 1-\mathrm{C} 4-\mathrm{C} 10$ & $78.5(3)$ & $\mathrm{C} 1-\mathrm{C} 4-\mathrm{C} 10-\mathrm{C} 5$ & $-3.0(4)$ \\
\hline $\mathrm{C} 10-\mathrm{C} 5-\mathrm{C} 6-\mathrm{C} 7$ & $-1.6(4)$ & $\mathrm{C} 5-\mathrm{C} 6-\mathrm{C} 11-\mathrm{C} 14$ & $5.8(4)$ \\
\hline $\mathrm{C} 10-\mathrm{C} 5-\mathrm{C} 6-\mathrm{C} 11$ & $176.5(3)$ & $\mathrm{C} 7-\mathrm{C} 6-\mathrm{C} 11-\mathrm{C} 14$ & $-176.1(3)$ \\
\hline
\end{tabular}




$\begin{array}{llll}\mathrm{C} 5-\mathrm{C} 6-\mathrm{C} 7-\mathrm{C} 8 & 1.4(4) & \mathrm{C} 5-\mathrm{C} 6-\mathrm{C} 11-\mathrm{C} 13 & 126.0(3) \\ \mathrm{C} 11-\mathrm{C} 6-\mathrm{C} 7-\mathrm{C} 8 & -176.8(3) & \mathrm{C} 7-\mathrm{C} 6-\mathrm{C} 11-\mathrm{C} 13 & -55.9(3) \\ \mathrm{C} 6-\mathrm{C} 7-\mathrm{C} 8-\mathrm{C} 9 & -0.2(5) & \mathrm{C} 5-\mathrm{C} 6-\mathrm{C} 11-\mathrm{C} 12 & -114.7(3) \\ \mathrm{C} 7-\mathrm{C} 8-\mathrm{C} 9-\mathrm{O} 1 & 179.2(2) & \mathrm{C} 7-\mathrm{C} 6-\mathrm{C} 11-\mathrm{C} 12 & 63.4(3) \\ \mathrm{C} 7-\mathrm{C} 8-\mathrm{C} 9-\mathrm{C} 10 & -0.9(5) & \mathrm{C} 3-\mathrm{C} 2-\mathrm{O} 1-\mathrm{C} 9 & -3.7(3) \\ \mathrm{C} 8-\mathrm{C} 9-\mathrm{C} 10-\mathrm{C} 5 & 0.7(4) & \mathrm{C} 8-\mathrm{C} 9-\mathrm{O} 1-\mathrm{C} 2 & -177.8(2) \\ \mathrm{O} 1-\mathrm{C} 9-\mathrm{C} 10-\mathrm{C} 5 & -179.4(2) & \mathrm{C} 10-\mathrm{C} 9-\mathrm{O} 1-\mathrm{C} 2 & 2.3(4) \\ \mathrm{C} 8-\mathrm{C} 9-\mathrm{C} 10-\mathrm{C} 4 & -178.9(3) & & \\ \mathrm{O} 1-\mathrm{C} 9-\mathrm{C} 10-\mathrm{C} 4 & 1.0(4) & & \end{array}$

Hydrogen-bond geometry $\left(A,{ }^{\circ}\right)$

\begin{tabular}{lllll}
\hline$D-\mathrm{H} \cdots A$ & $D-\mathrm{H}$ & $\mathrm{H} \cdots A$ & $D \cdots A$ & $D-\mathrm{H} \cdots A$ \\
\hline $\mathrm{C} 3-\mathrm{H} 3 \cdots \mathrm{O} 2^{\mathrm{i}}$ & 0.95 & 2.42 & $3.334(4)$ & 162
\end{tabular}

Symmetry code: (i) $x,-y+1 / 2, z-1 / 2$. 